\title{
Phenotypic research on senile osteoporosis caused by SIRT6 deficiency
}

\author{
De-Mao Zhang, Di-Xin Cui, Ruo-Shi Xu, Ya-Chuan Zhou, Li-Wei Zheng, Peng Liu and Xue-Dong Zhou
}

Osteoporosis is a serious public bone metabolic disease. However, the mechanisms underlying bone loss combined with ageing, which is known as senile osteoporosis, remains unknown. Here we show the detailed phenotype of this disease caused by SIRT6 knock out (KO) in mice. To the best of our knowledge, this is the first study to reveal that SIRT6 is expressed in both bone marrow stroma cells and bone-related cells in both mouse and human models, which suggests that SIRT6 is an important regulator in bone metabolism. SIRT6-KO mice exhibit a significant decrease in body weight and remarkable dwarfism. The skeleton of the SIRT6-KO mouse is deficient in cartilage and mineralized bone tissue. Moreover, the osteocalcin concentration in blood is lower, which suggests that bone mass is markedly lost. Besides, the tartrate-resistant acid phosphatase 5b (TRAP5b) concentration is much higher, which suggests that bone resorption is overactive. Both trabecular and cortical bones exhibit severe osteopenia, and the bone mineral density is decreased. Moreover, double-labelling analysis shows that bone formation is much slower. To determine whether SIRT6 directly regulates bone metabolism, we cultured primary bone marrow stromal cells for osteogenesis and osteoclastogenesis separately to avoid indirect interference in vivo responses such as inflammation. Taken together, these results show that SIRT6 can directly regulate osteoblast proliferation and differentiation, resulting in attenuation in mineralization. Furthermore, SIRT6 can directly regulate osteoclast differentiation and results in a higher number of small osteoclasts, which may be related to overactive bone resorption.

International Journal of Oral Science (2016) 8, 84-92; doi:10.1038/ijos.2015.57; published online 29 April 2016

Keywords: ageing; osteoclastogenesis; osteogenesis; osteoporosis

\section{INTRODUCTION}

Osteoporosis is a serious public disease, particularly in China, as China's society is increasingly ageing. ${ }^{1}$ Globally, osteoporosis is the most common bone metabolic disease of the elderly. ${ }^{2}$ There are two subtypes of osteoporosis: type I is called postmenopausal osteoporosis or oestrogen-deficient osteoporosis, and often occurs after female menopause due to a decline in oestrogen levels. Type II is senile osteoporosis (SOP), which usually occurs in females at 65 years of age or in males at 70 years of age. Although great progress has been made in understanding the mechanism underlying oestrogen-deficient osteoporosis within the past several years, it remains unknown how bone loss is caused by ageing, which may be correlated to SIRT6-based mechanisms according to previous research. ${ }^{3}$ Age-related bone loss is associated with ageing in both genders, and there is a greater decrease in bone formation by osteoblasts and a significant increase in bone resorption by osteoclasts, ${ }^{4}$ which consequently results in osteoporosis.

Recent studies have used functional genetic approaches and gene knock out (KO) technology as a new method to study the development of many biological problems in the process of ageing. It is well known that the family of deacetylase sirtuin (SIRT1-7) is involved in ageing and age-related diseases, which provides a new approach to the study of biological diseases, including bone loss in ageing. ${ }^{3}$ It has been reported that the SIRT family is involved in a broad range of biological processes such as DNA repair, neurological protection, and body metabolism. The most recent study has shown that SIRT6, an nicotinamide adenine dinucleotide (NAD)-dependent deacetylase, has a critical role in anti-ageing. ${ }^{5-6}$ SIRT6 functions as a deacetylase and nucleotidyltransferase, which removes chemicals from acetyl groups from a specific site of the histone, thereby regulating specific gene expression. As reported, SIRT6-deficient mice exhibit a short lifespan and multiple organ ageing, including reduced blood glucose and insulin-like growth factor (IGF) concentrations, increased DNA instability, and bone loss at an early period..$^{5}$ As a histone-H3 lysine deacetylase, SIRT6 can regulate the stability of telomere chromatin and the genetic expression of the nuclear factor-kappa B signalling pathway, which affects lifespan. ${ }^{7}$ SIRT6 is also involved in various biological processes such as inflammation, metabolic balance, and even cancer metabolism in addition to ageing, as the liver-specific deletion of SIRT6 in mice results in fatty liver formation. ${ }^{8}$ SIRT6 is a master regulator of glucose homeostasis and may have a large role in the treatments of metabolic diseases such as diabetes and obesity. ${ }^{9}$ 
Moreover, SIRT6 is a potent tumour suppressor and acts to suppress cancer metabolism. ${ }^{10}$

A previous study has demonstrated that SIRT6-deficient mice exhibits $\sim 30 \%$ bone mineral density (BMD) loss and die at $\sim 4$ weeks of age. Rapid bone loss develops closely and synchronously with the accelerated ageing process, and such an osteoporotic phenotype is an excellent model for the study of ageing-associated osteoporosis. ${ }^{3}$ However, it remains unknown how SIRT6 regulates the progress of bone metabolism and how SIRT6 induces bone loss during the ageing progress. In this study, we used SIRTO-KO mice to investigate the bone pathological changes with ageing progression. Given the emerging roles of SIRT6 in anti-ageing, we demonstrated a smaller somatotype and decreased bone density in SIRT6-KO mice.

\section{MATERIALS AND METHODS}

Experimental animals and analysis of the skeletal phenotype All mouse experiments were performed according to the approved protocols by the Institutional Animal Care and Use Committees at Sichuan University. SIRT6 global KO mice (129/SvJ) were purchased from Jackson Laboratory (Bar Harbor, ME, USA). Collagen type I $\alpha 1$-green fluorescent protein (ColI $\alpha 1$-GFP) transgenic mice were also purchased from Jackson Laboratory, and they expressed the topaz variant of green fluorescent protein (GFP) under the control of rat ColI $\alpha 1$ promoter/enhancer sequence. We crossed ColI $\alpha 1-$ GFP mice with heterozygous SIRT6 mice several times until homozygosity for ColI $\alpha 1-$ GFP was achieved, and GFP expression in the bone correlated with new bone mass. We used male mice (3 weeks old) in our studies. For skeleton staining, 3-week-old mice were eviscerated, and the skin was carefully removed. After $18 \mathrm{~h}$ of fixation in $95 \%$ ethanol, samples were stained in an Alcian blue solution (150 mg Alcian blue, $800 \mathrm{~mL}$ $95 \%$ ethanol, and $500 \mathrm{~mL}$ acetic acid) for $24 \mathrm{~h}$. Next, the animals were transferred to $2 \% \mathrm{KOH}$ for $48 \mathrm{~h}$. The skeletons were then stained in Alizarin red solution $\left(30 \mathrm{mg} \cdot \mathrm{L}^{-1}\right.$ Alizarin red in $\left.2 \% \mathrm{KOH}\right)$ and cleared in $1 \% \mathrm{KOH} / 20 \%$ glycerol.

\section{Histology and bone histomorphometry}

Decalcified and non-decalcified sections of bone were prepared as previously described. ${ }^{11-12}$ Briefly, mice (2 weeks old) were injected subcutaneously with calcein $\left(8 \mathrm{mg} \cdot \mathrm{kg}^{-1}\right.$; Sigma-Aldrich Biotechnology, St Louis, MO, USA) 4 days after they had received a xylenol orange (XO; $15 \mathrm{mg} \cdot \mathrm{kg}^{-1}$; Sigma-Aldrich Biotechnology, St Louis, MO, USA) injection. Samples were collected when the mice were 3 weeks old. Next, the samples were fixed with $4 \%$ paraformaldehyde overnight at $4{ }^{\circ} \mathrm{C}$. Non-decalcified bones were embedded in optimal cutting temperature (OCT) compound, and 8 - $\mu \mathrm{m}$-thick frozen sections were prepared. Decalcified bones were embedded in paraffin, and 5- to 7$\mu \mathrm{m}$-thick sections were prepared. For micro-computed tomography $(\mu \mathrm{CT})$ analysis, we performed high-resolution $\mu \mathrm{CT}$ scanning ( $\mu \mathrm{CT} 50$; Scanco, Bassersdorf, Zurich, Switzerland) to measure the morphological indices of the metaphyseal regions of the femur or lumbar vertebrae (L5-6; 3 weeks old). ${ }^{12-13} \mu \mathrm{CT}$ imaging analysis of the metaphyseal regions was performed with 70 slices $(10 \mu \mathrm{m}$ per slice). The threshold is was $150 \mathrm{mg} \cdot \mathrm{cm}^{-1}$. The expression of SIRT6 was determined using immunohistochemistry, according to the manufacturer's instructions (Vector Laboratories, Burlingame, CA, USA).

\section{Enzyme-linked immunosorbent assay}

Test samples consisted of fresh mouse plasma. Enzyme-linked immunosorbent assay kits were purchased for TRAP5b and osteocalcin $(\mathrm{OCN})$ assays, and were performed according to the manufacturer's protocols (Catalogue No. SEA902Mu and SEA471Mu, Cloud Clone, Wuhan, China).

\section{Mouse bone marrow stromal cell culture}

Primary bone marrow stromal cells (BMSCs) were cultured using bone marrow obtained from mouse femurs and tibia as previously described. ${ }^{14}$ Briefly, mouse bone marrow was flushed from the femora and tibia, and cultured in $\alpha$-modified Eagle's minimal essential medium ( $\alpha$-MEM; Catalogue No. SH30265; Hyclone Laboratories, Logan, UT, USA), which was supplemented with $10 \%$ fetal bovine serum (Hyclone Laboratories, Logan, UT, USA) and 1\% penicillin/ streptomycin (Life Technologies, Gaithersburg, MD, USA). The cell density was $\sim 20 \times 10^{6}$ per $\mathrm{mL}$. For osteogenic differentiation, cells were cultured in basal medium for 5 days and were then changed to "osteogenic differentiation medium" ( $\alpha$-MEM supplemented with $10^{-8} \mathrm{~mol} \cdot \mathrm{L}^{-1}$ dexamethasone, $8 \mathrm{mmol} \cdot \mathrm{L}^{-1} \beta$-glycerophosphate, and $50 \mu \mathrm{g} \cdot \mathrm{mL}^{-1}$ ascorbic acid) for an additional 14 days.

For XO labelling, cells were cultured in medium with $40 \mathrm{mmol} \cdot \mathrm{L}^{-1}$ $\mathrm{XO}$ overnight. Images of the cells were obtained using a fluorescence microscope (green for ColI $\alpha 1$-GFP, blue for 4',6-diamidino-2-phenylindole (DAPI), and red for XO). For alkaline phosphatase (ALP) staining, the medium was discarded, and the cells were fixed and washed twice with phosphate-buffered saline. Next, the cells were stained with ALP staining solution, which consisted of naphthol AS-MX phosphate and fast red violet LB salt (Sigma-Aldrich Biotechnology, St Louis, MO, USA). Finally, the stained wells were washed twice with tap water and imaged. For Von Kossa and Van Gieson staining, cells were stained using Von Kossa staining solution and Van Gieson staining solution, independently. Finally, the stained wells were washed twice with tap water and imaged.

The osteoclast cultures were generated as previously described. ${ }^{15}$ Briefly, bone marrow haematopoietic cells were cultured in the presence of macrophage colony-stimulating factor (M-CSF; $10 \mu \mathrm{g} \cdot \mathrm{mL}^{-1}$ for 2 days), and incubated with both M-CSF and receptor activator of nuclear factor- $\kappa \mathrm{B}$ ligand (RANKL; $10 \mu \mathrm{g} \cdot \mathrm{mL}^{-1}$ ) for 5 days. Next, the cell medium was discarded, and the cells were fixed for $5 \mathrm{~min}$ and then stained for tartrate-resistant acid phosphatase (TRAP). TRAP-positive cells with more than two nuclei were quantified as osteoclasts.

\section{Statistical analysis}

The positively stained area was calculated using the Image-Pro Plus software (IPP version 6.0; Media Cybernetics, Carlsbad, CA, USA). All experimental results were obtained from at least four separate experiments. Student's $t$-test analysis of variance was performed on the data using SPSS version 16.0 (SPSS, Chicago, IL, USA). Values were considered significantly different if $P<0.05$.

\section{RESULTS}

\section{Endogenous expression of SIRT6 in bone tissue}

To investigate the basal expression of SIRT6 in bone tissue, we collected human rib bones and mouse femora, and examined these samples using paraffin section immunohistochemistry. SIRT6 was expressed in osteocytes, osteoblasts (along the surface of the mineralized tissue), and many types of marrow stroma cells of human rib bones, as well as the mouse femora (Figure 1). These results indicated that the positive expression of SIRT6 might participate in bone metabolism. 

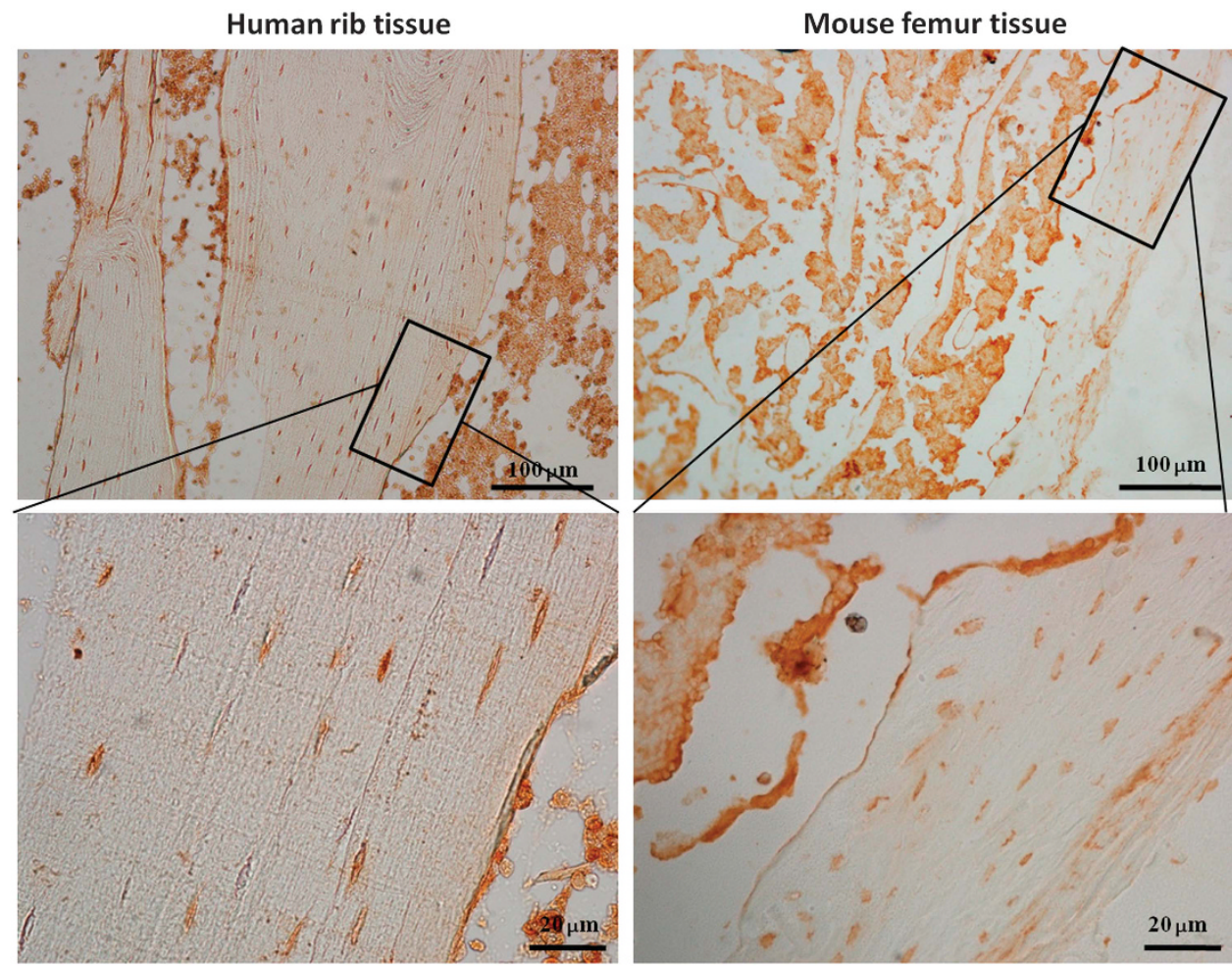

Figure 1 Expression of SIRT6 in bone tissue of both mouse and human using immunohistochemistry staining. The images are obtained from rib tissue of human and femur of wild-type mouse.

a

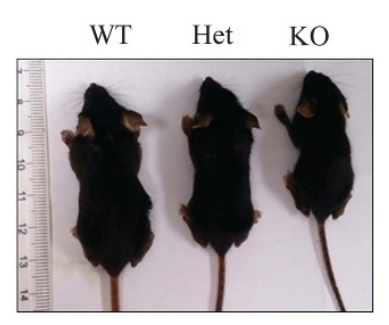

d

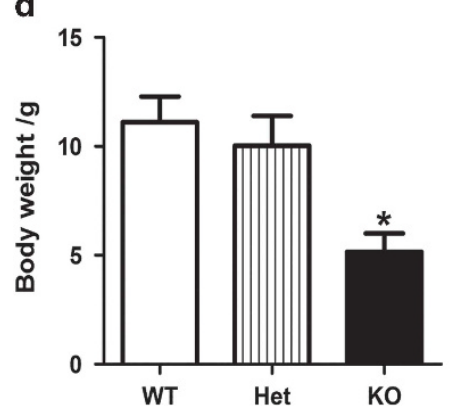

b

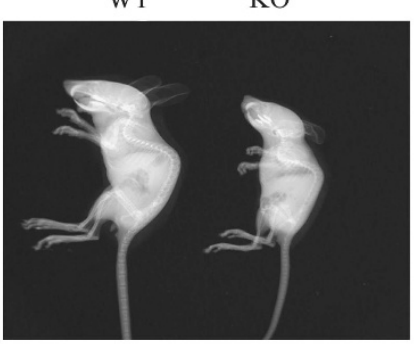

e

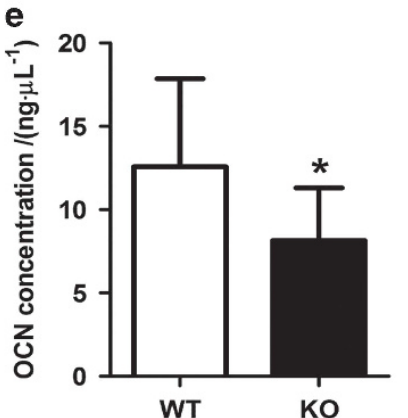

c

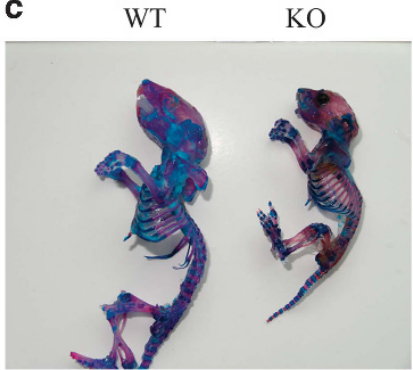

$f$

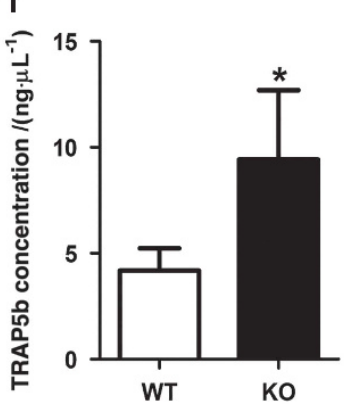

Figure 2 SIRT6 knock out mice. (a, d) Body weight was determined in 9-11 mice for each group. (b) X-ray image. (c) Alizarin red S- and Alcian blue staining for skeletons. (e, f) Osteocalcin and TRAP5b concentration in blood was analyzed using enzyme-linked immunosorbent assay (ELISA) with 11-12 mice in each group. ${ }^{*} P<0.05$. Het, heterozygote; KO, knock out; OCN, osteocalcin; WT, wild type.

Skeleton development analysis on SIRT6-KO mice

We successfully bred and divided male littermate mice at the age of 3 weeks into groups consisting of wild type (WT), SIRT6 heterozygote and SIRT6-KO mice according to genotype identification analysis. On the basis of previous research on ageing SIRT6-KO mice, ${ }^{3}$ the entire body skeleton was investigated in this study. Overall, SIRT6-KO mice exhibited remarkable dwarfism and weight loss compared with their WT littermates, but no significant difference was observed between WT and SIRT6 heterozygote mice (Figure 2a-2d). Furthermore, Alizarin red S- and Alcian blue staining was performed to observe 


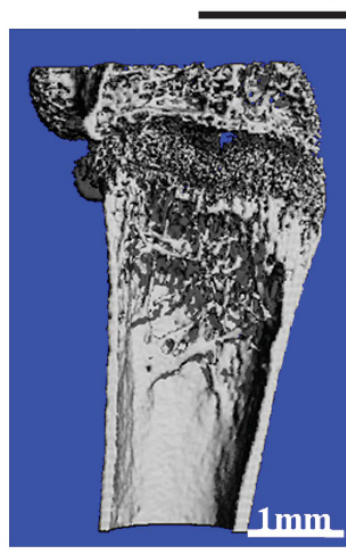

WT
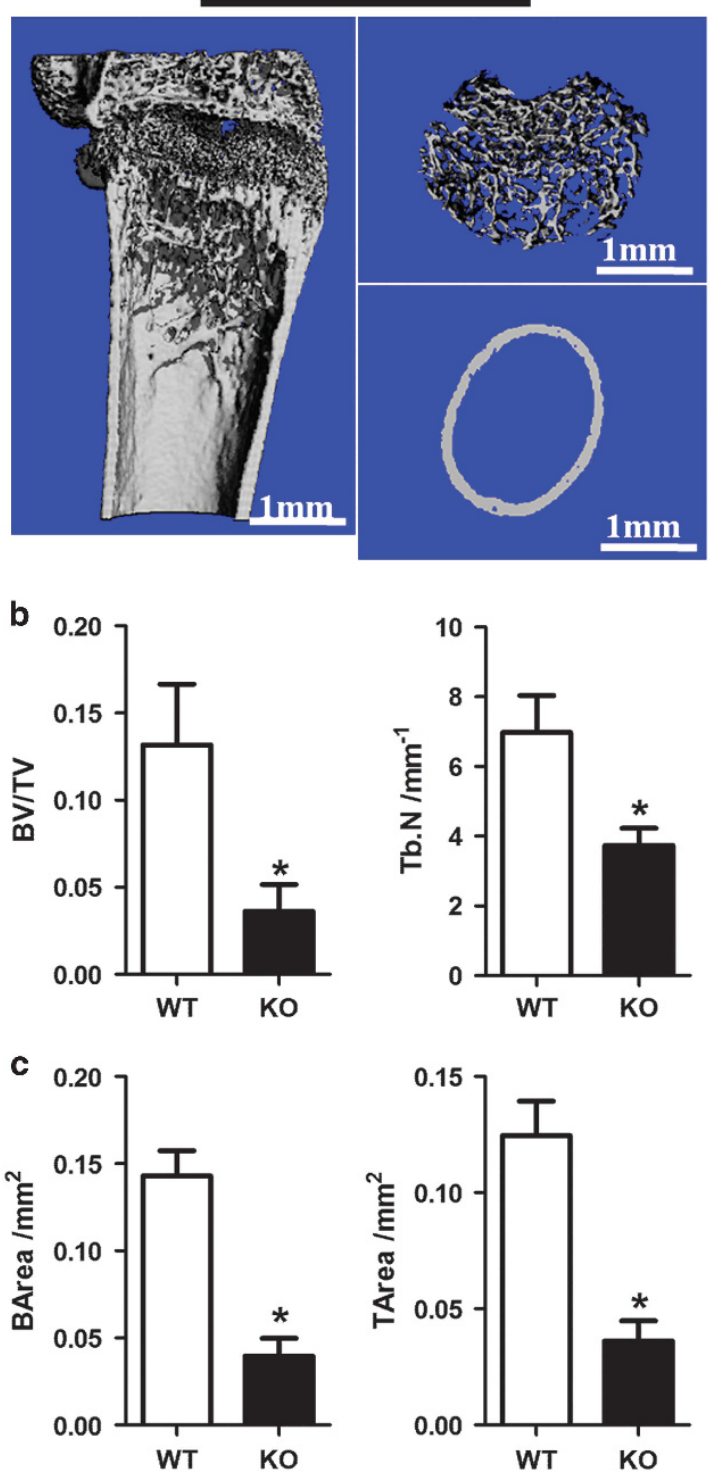
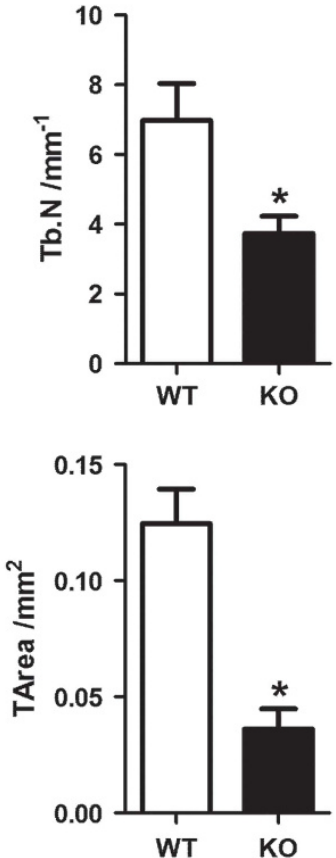
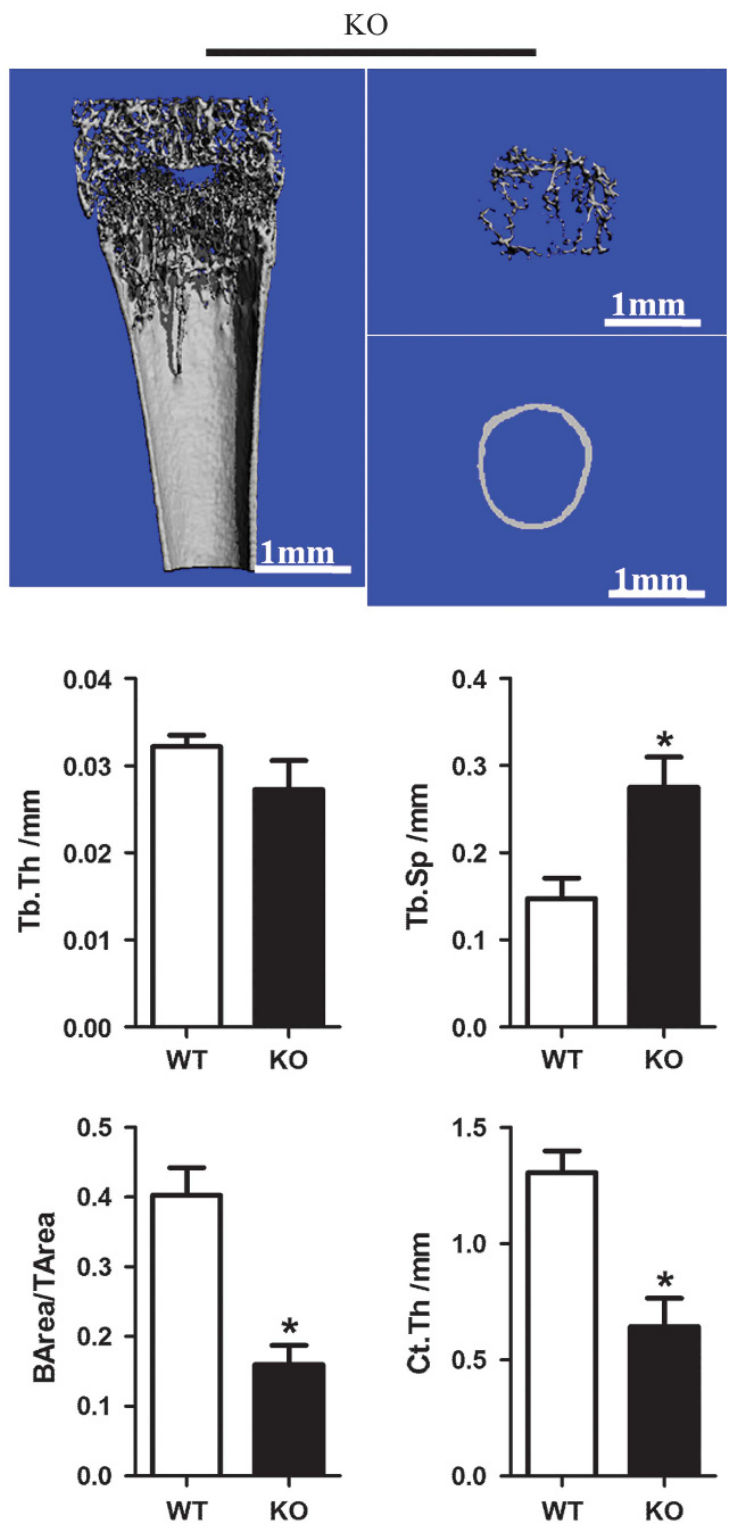

Figure 3 Micro-CT analysis of trabecular and cortical bone of the femur. (a) Femur tissue. Trabecular bone is from the part nearby the metaphysical area. Cortical bone is from mid-shaft. (b, c) Representative parameters of trabecular and cortical bone, respectively. ${ }^{*} P<0.05$. There were seven mice in each group used for the statistical analyses. BArea, bone area; BV/TV, bone volume/the total volume; CT, computed tomography; Ct.Th, cortical bone thickness; KO, knock out; TArea, the total area; Tb.N, trabecular number; Tb.Sp, trabecular separation; Tb.Th, trabecular thickness; WT, wild type.

alterations in the entire body skeleton structure (Figure 2c). Compared with the WT littermates, cartilage bone shown as blue staining in SIRT6-KO mice was significantly decreased. In addition, lower calcification of the long bone was also observed when using red staining in SIRT6-KO mice. Moreover, X-ray analysis for the skeleton indicated that the whole-body BMD of SIRT6-KO mice was decreased, which was consistent with the findings obtained in previous work. ${ }^{3}$ Furthermore, to investigate the condition of whole-body bone (Figure 2b) metabolism, we collected serum from WT and SIRT6$\mathrm{KO}$ mice to examine the expression of some markers of bone remodelling. The OCN (a marker of bone mass) concentration in serum was significantly decreased, whereas the TRAP5b concentration was significantly increased (Figure $2 \mathrm{e}$ and $2 \mathrm{f}$ ), which indicated bone mass loss and bone resorption increase. Overall, SIRT6 deficiency in mice resulted in skeletal dysplasia, BMD decrease, and bone resorption increase of whole body bone tissue.
Bone histomorphometry was performed using $\mu \mathrm{CT}$ analysis, which further demonstrated bone growth retardation and severe osteopenia in SIRT6-KO mice. Trabecular bone mass formation was significantly impaired, with a reduced trabecular bone volume per tissue volume and a reduced trabecular number. The trabecular space was concomitantly increased (Figure $3 \mathrm{a}$ and $3 \mathrm{~b}$ ). Moreover, the cortical bone thickness of SIRT6-KO mice was decreased, as observed in the longitudinal and transverse mid-diaphysis sections. The parameters of cortical bone, cortical bone area, total cross-sectional area, cortical area fraction (BArea/TArea), and average cortical thickness were all decreased (Figure 3a-3c). However, histological examination showed a size difference in the femora of SIRT6-KO mice. A significantly reduced trabecular bone mass near the femur growth plate was demonstrated in haematoxylin and eosin (H\&E) staining sections. Furthermore, the diaphyseal cortical bone was thinner than that of WT mice (Figure 4a). Von Kossa staining presented less calcium 
a
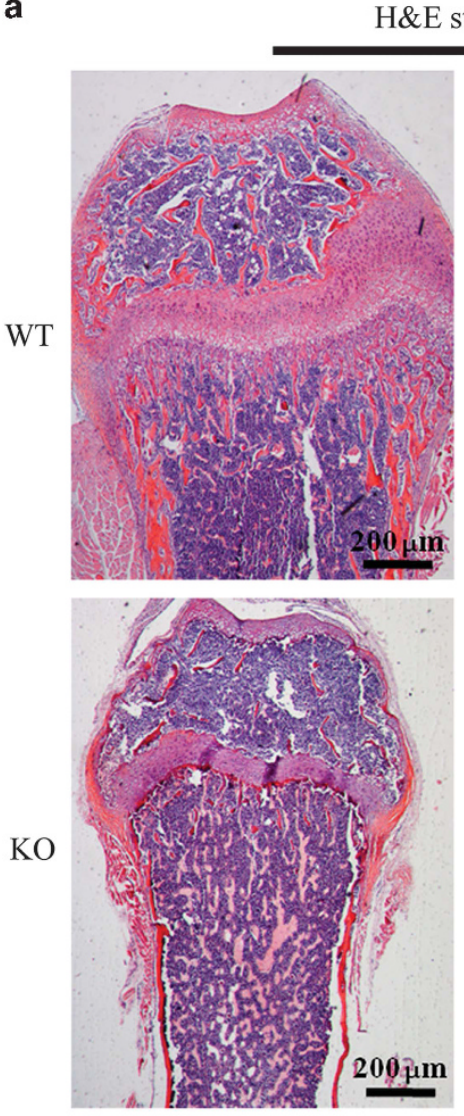

c b

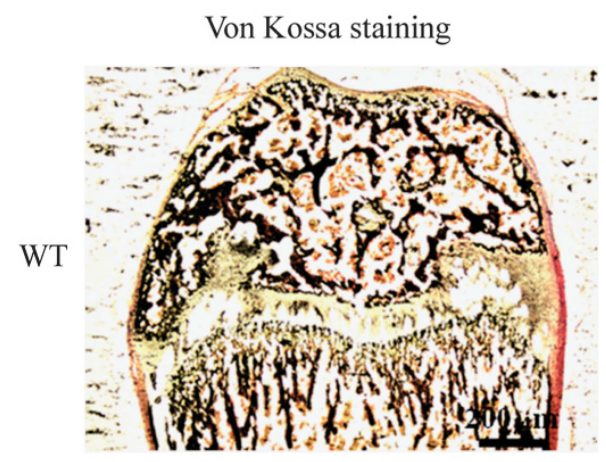

$\mathrm{KO}$

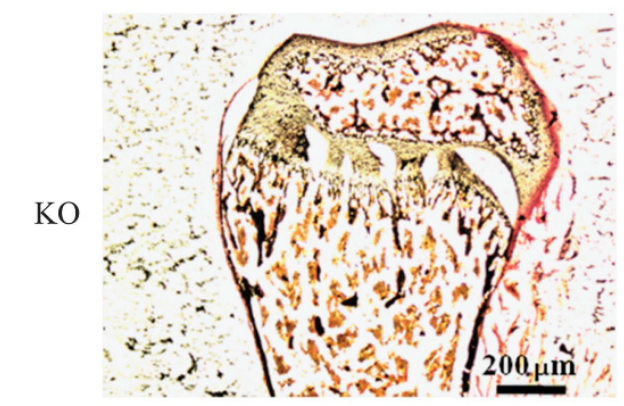

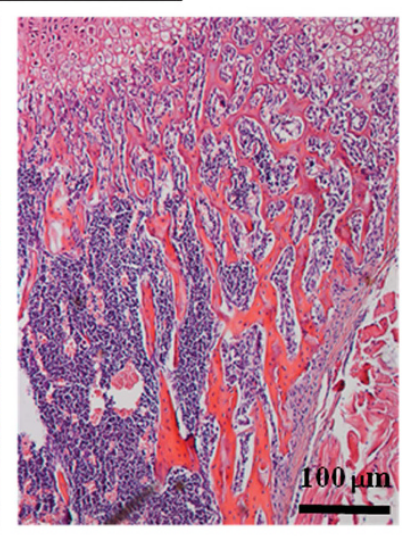

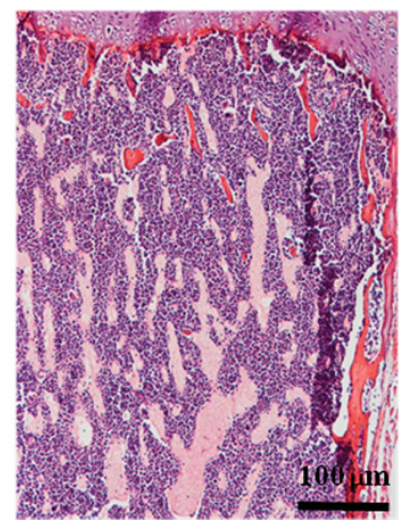

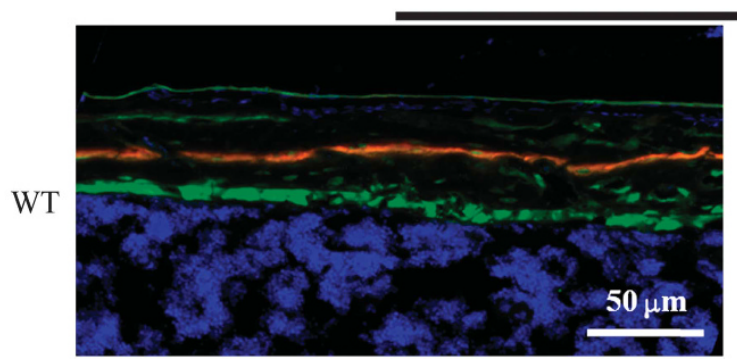

Double labeling

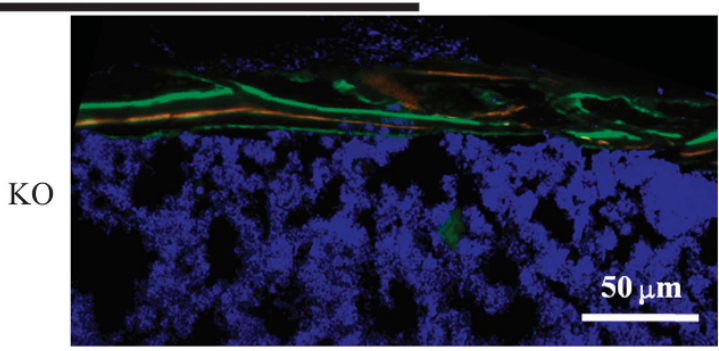

Figure 4 Histological examination. (a) Haematoxylin and eosin staining of the femur nearby the metaphysic area. (b) Van Gieson (red) and Von Kossa (black) staining for the mineralization of bone tissue. (c) Double-labelling analysis for bone formation at the femoral mid shaft. The red line is marked using xylenol orange, while the green line is marked by calcein. H\&E, haematoxylin and eosin; KO, knock out; WT, wild type.

deposition with less black mineralized bone tissue in the SIRT6KO mouse femora (Figure 4b). Double fluorochrome labelling revealed a reduction in the distance between green (stained by calcein) and red (stained by XO) in SIRT6-KO mice, which indicated a lower bone formation rate and was suggestive of osteogenic activity (Figure 4c). These results presented severely impaired bone metabolism due to SIRT6 KO, which is a clear symptom of osteoporosis.

\section{Osteogenesis in SIRT6-KO mice}

To further investigate whether SIRT6 directly regulates bone formation, we cultured primary BMSCs for osteogenesis in vitro (Figures 5 and 6). Interestingly, ALP production, which is an early marker of osteogenesis, was slightly increased at an early stage of osteogenesis (Figure 5). However, it was markedly decreased during the late stage of osteogenesis at day 21 (Figure 6). Similarly, ColI $\alpha 1$-GFP was observed with increased expression at day 7 , but markedly decreased expression was observed at day 21. Fewer calcified nodules were observed using phase contrast microscopy in SIRT6-KO BMSCs. This condition of mineralization was also detected using XO and Von Kossa staining simultaneously, which further demonstrated significantly decreased osteogenesis. These results, which were consistent with our observations in vivo, revealed that SIRT6 could directly regulate bone formation.

\section{Osteoclastogenesis in SIRT6-KO mice}

Osteoclastogenesis is also an important aspect of bone metabolism. ${ }^{6,16}$ Thus, we wondered whether SIRT6 directly regulated osteoclastogenesis. We cultured primary BMSCs from WT and SIRT6-KO mice that were stimulated with M-CSF and RANKL for osteoclast induction. On day 7, TRAP staining revealed that there 
a

홍
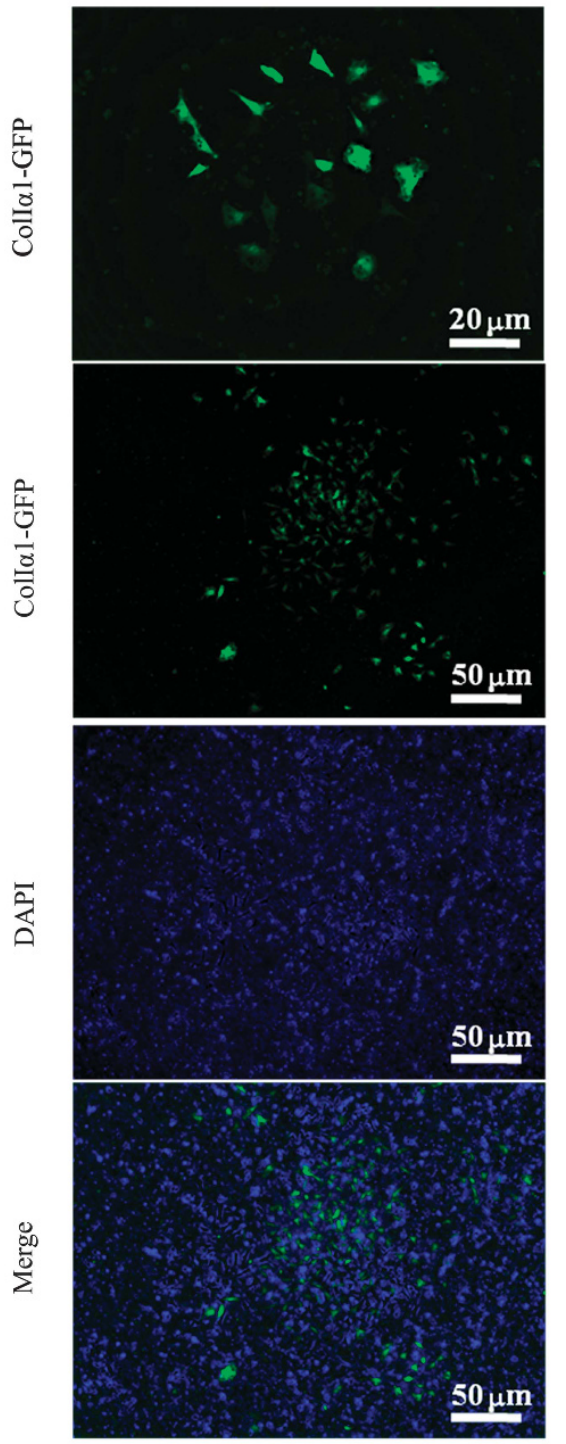

b

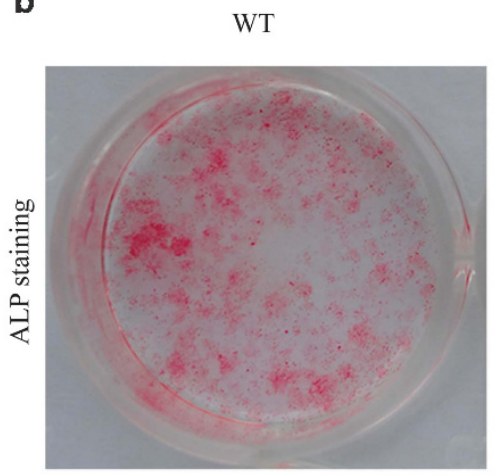

$\mathrm{KO}$

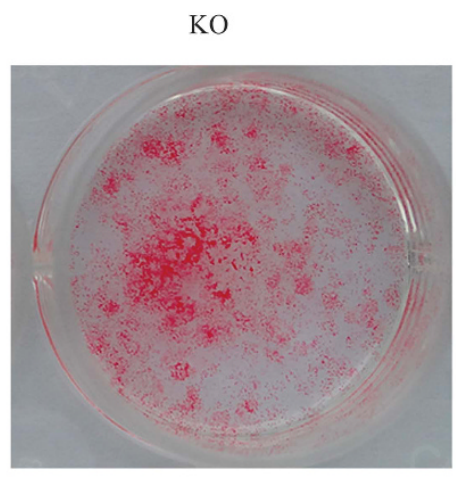

$\mathrm{KO}$
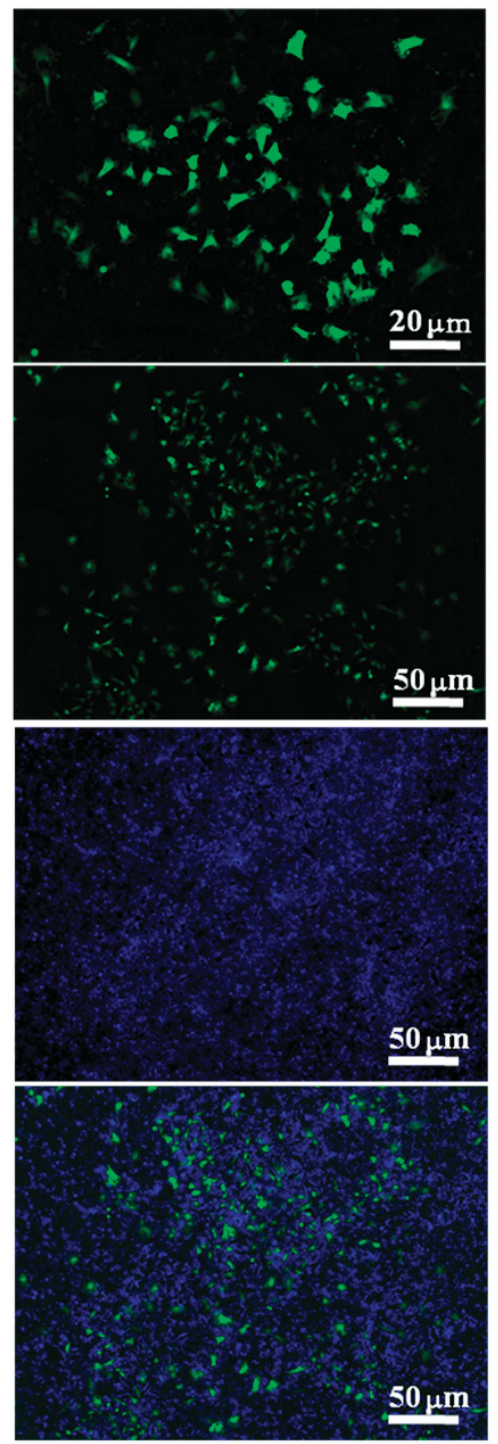

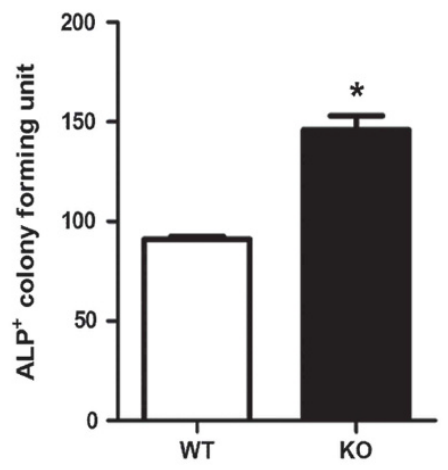

Figure 5 Day 7 of cell culture for osteoblastogenesis from bone marrow stromal cells. (a) ColI $\alpha 1$-GFP expression. (b) ALP for the cell colony forming units. The data are representative of six independent experiments. ${ }^{*} P<0.05$. ALP, alkaline phosphatase staining; DAPI, 4',6-diamidino-2-phenylindole; GFP, green fluorescent protein; KO, knock out; WT, wild type.

were fewer calcified mature giant osteoclasts with multi-nuclei and foamy cytoplasm in the SIRT6-KO group. However, a significant increase in TRAP+ individual osteoclasts with more than two nuclei was observed in SIRT6-KO osteoclasts (Figure 7). We proposed that cell fusion at the late stage of osteoclast formation might have been disrupted by SIRT6 deficiency, thereby inhibiting 
a
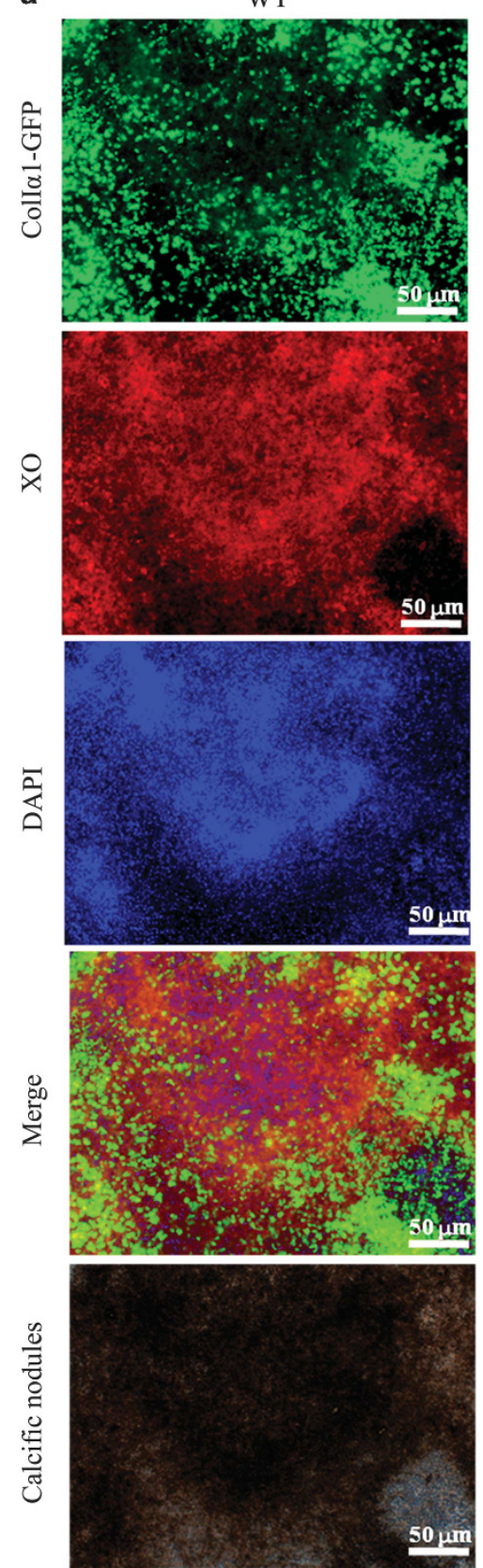

b

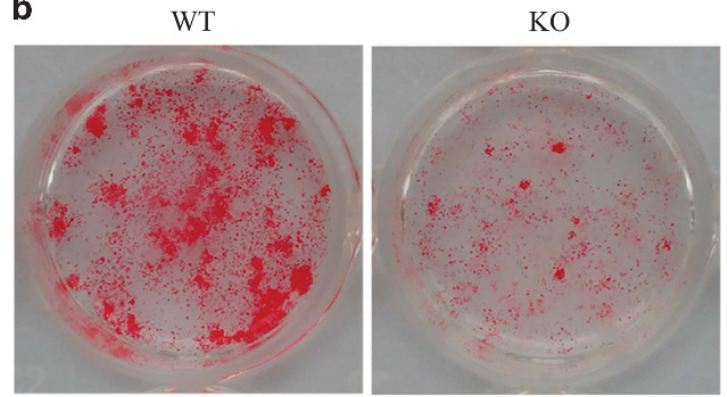

ALP staining
$\mathrm{KO}$

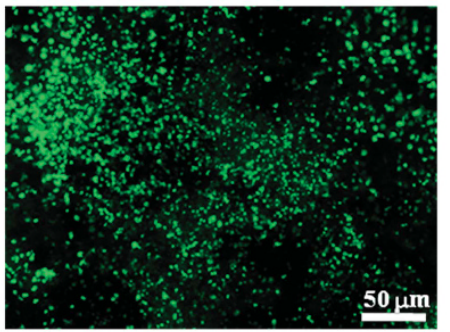

$50 \mu \mathrm{m}$
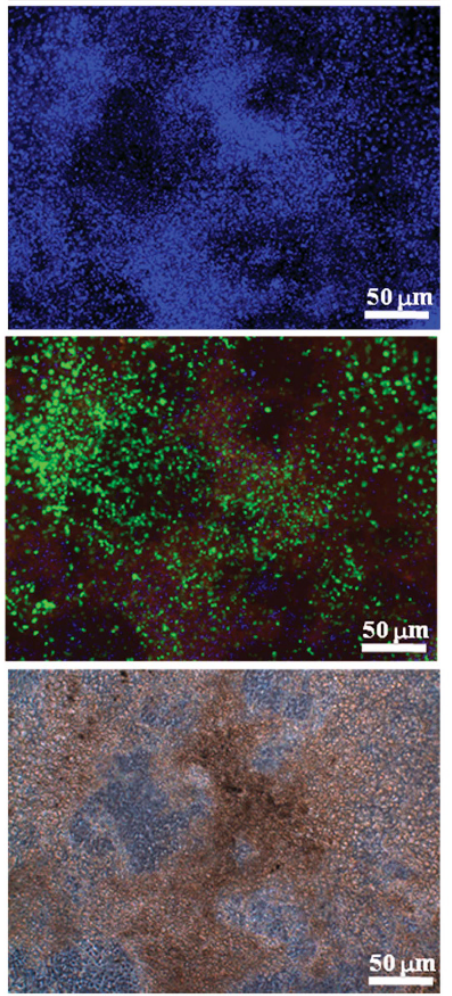

c
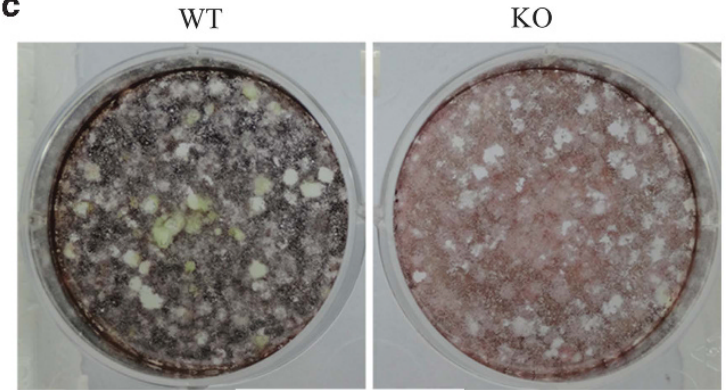

Von Kossa staining

Figure 6 Day 21 of cell culture for osteoblastogenesis. (a) Expression of ColI $\alpha 1-G F P$, xylenol orange fluorescence for calcification and calcific nodules condition. (b, c) Alkaline phosphatase staining and Von Kossa staining. ALP, alkaline phosphatase; DAPI, 4',6-diamidino-2-phenylindole; GFP, green fluorescent protein; KO, knock out; WT, wild type; XO, xylenol orange. 
a
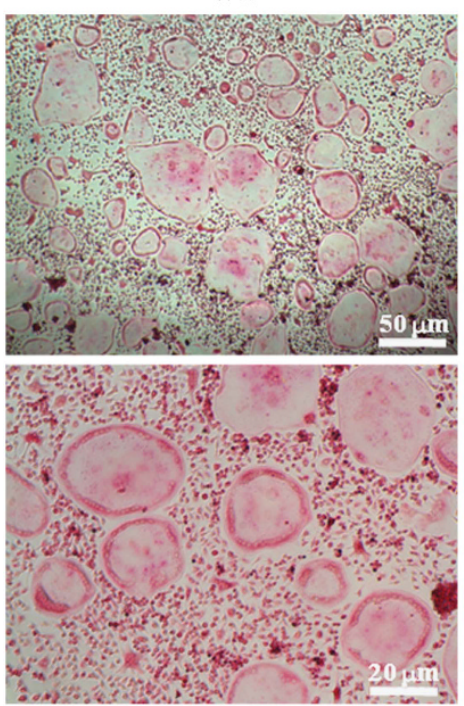
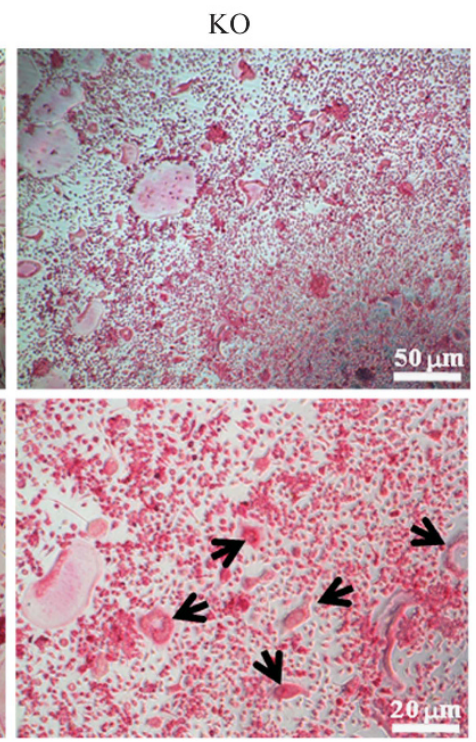

b

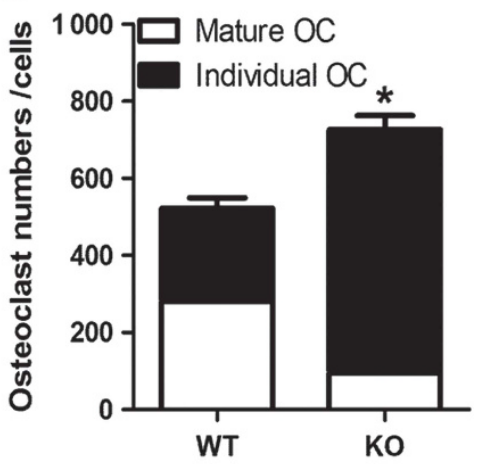

Figure 7 Day 7 of cell culture for osteoclastogenesis. (a) Trap staining for osteoclast. (b) The cell number of osteoclast per well. The 48-well plates were used for cell culture. Large cells with multi-nuclei and foamy cytoplasm were quantified as mature osteoclasts. Other Trap+ but smaller cells with more than two nuclei were marked as individual osteoclasts, such as the cells indicated by black arrows in figure. The data are representative of six independent experiments. ${ }^{*} P<0.05$. KO, knock out; OC, osteoclast; Trap, tartrate-resistant acid phosphatase; WT, wild type.

the maturation process. Taken together, these results indicated that SIRT6 has a role in directly controlling the formation, morphology, and number of mature osteoclasts.

\section{DISCUSSION}

In this study, SIRT6-KO mice were proven to be a good model for the study of SOP disease. It has been reported that SIRT6-KO mouse is an ageing model. ${ }^{3,17}$ On the basis of this research, we aimed to fully investigate the phenotype of bone metabolism in detail and demonstrate its involvement in severe osteoporosis, which is ageing-related.

Generally, bone formation begins in mesenchymal cells from the bone marrow, which differentiate into osteoblasts that are attached to the bone surface and, consequently, differentiate into mature osteocytes, which are buried inside the calcified tissue. ${ }^{6,18}$ Basal expression of SIRT6 was found in bone marrow and mature osteocytes in both mouse and human models (Figure 1), thereby suggesting that SIRT6 is likely to participate in the entire process of bone formation. The deterioration in both body length and weight and the reduction of cartilage and mineralized bone (Figure 2) were similar to the manifestation of osteopenia in aged people. Trabecular bone in the metaphyseal area and cortical bone in the diaphysis was reduced, as shown in $\mu \mathrm{CT}$ analyses, and converted into a status that appears weaker than the normal (Figure 3). Scattered trabecular bone detected using $\mathrm{H} \& \mathrm{E}$ and Von Kossa analysis revealed that bone metabolism of SIRT6-KO mice was disrupted, which was also shown by a lower bone formation rate (Figure 4) and by markers that were altered in blood, indicating wholebody bone metabolism (Figure 2). To a greater degree, most of the detailed phenotypes of osteopenia caused by SIRT6 KO are presented in our work, which describes the symptom of SOP disease.

We further investigated SOP at the cellular level in vitro to identify SOP results from weakened osteogenesis and enhanced bone resorption caused by SIRT6 KO directly. Bone-related features are an integrative effect that needs to be differentiated into osteogenesis and bone resorption.1,19 ELISA of the blood of SIRT6-KO mice revealed decreased OCN and increased TRAP5b (Figure 2), which demonstrated that bone activity featuring SIRT6 deficiency was affected by both bone formation and bone resorption. Thus, bone loss in SIRT6-KO mice resulted from an imbalance of osteogenesis and bone resorption. Double labelling of bone revealed a conspicuous performance of sluggish new bone growth progression (Figure 4). Isolated and differentiated osteoblasts were weak in collagen formation and calcification, as shown in Figure 5, whereas isolated and differentiated osteoclasts were strong in bone resorption, as shown in bone slices (Figure 7). With less new bone formed and more bone resorbed in SIRT6-KO mice, the balance of the bone condition tilts naturally to bone loss.

Interestingly, osteogenic induction experiments showed a slight increase in ALP and ColI $\alpha 1-$ GFP in BMSCs at the early stage of 7 days, whereas both decreased and the staining was reduced at the late stage (Figure 6). Sugatani et al. ${ }^{20-22}$ obtained similar results with SIRT6-KO BMSCs, which may be explained by the increased expression of Runx2 in osteoblasts. ${ }^{20-22}$ In all, SIRT6 defects further support the idea that SIRT6 leads to low calcification and exacerbates senile osteoporosis to some degree. Furthermore, we are interested in continuing our research to obtain more detailed evidence on the mechanism of SOP disease with SIRT6 deficiency.

\section{ACKNOWLEDGEMENTS}

This study was supported by NSFC grants 81371136 and JCPT2011-9 (Xue-Dong Zhou), and NSFC grants 81470711 and 81200760 (Li-Wei Zheng). We thank Ling Ye (Professor, Sate Key Laboratory of Oral Diseases, West China Hospital of Stomatology, Sichuan University) for financial aid, and many thanks to Rui-Min Li, Feng Lou, Ming-Ru Bai, Ke Wang, Yi-Lin Ping, Fan-Yuan Yu, Fan-Zi Wu and Xiao Yang for valuable help. 
1 Sims NA, Martin TJ. Coupling the activities of bone formation and resorption: a multitude of signals within the basic multicellular unit. Bonekey Rep 2014; 3: 481.

2 Shuler FD, Conjeski J, Kendall D et al. Understanding the burden of osteoporosis and use of the World Health Organization FRAX. Orthopedics 2012; 35(9): 798-805.

3 Mostoslavsky R, Chua KF, Lombard DB et al. Genomic instability and aging-like phenotype in the absence of mammalian SIRT6. Cell 2006; 124(2): 315-329.

4 Feng X, McDonald JM. Disorders of bone remodeling. Annu Rev Pathol Mech Dis 2011; 6(1): 121-145

5 Lombard DB, Schwer B, Alt FW et al. SIRT6 in DNA repair, metabolism and ageing. J Intern Med 2008; 263(2): 128-141.

6 Sims NA, Gooi JH. Bone remodeling: multiple cellular interactions required for coupling of bone formation and resorption. Semin Cell Dev Biol 2008; 19(5): 444-451.

7 Kawahara TL, Michishita E, Adler AS et al. SIRT6 links histone H3 lysine 9 deacetylation to NF-кB-dependent gene expression and organismal life span. Cell 2009; 136(1): 62-74.

8 Kim HS, Xiao C, Wang RH et al. Hepatic-specific disruption of SIRT6 in mice results in fatty liver formation due to enhanced glycolysis and triglyceride synthesis. Cell Metab 2010; 12(3): 224-236.

9 Zhong L, D'Urso A, Toiber D et al. The histone deacetylase Sirt6 regulates glucose homeostasis via Hif1alpha. Cell 2010; 140(2): 280-293.

10 Sebastián C, Zwaans BM, Silberman DM et al. The histone deacetylase SIRT6 is a tumor suppressor that controls cancer metabolism. Cell 2012; 151(6): 1185-1199.

11 van't Hof RJ, Clarkin CE, Armour KJ. Studies of local bone remodeling. The calvarial injection assay. Methods Mol Med 2003; 80: 345-351.

12 Zhu LL, Blair H, Cao J et al. Blocking antibody to the $\beta$-subunit of FSH prevents bone loss by inhibiting bone resorption and stimulating bone synthesis. Proc Natl Acad Sci USA 2012; 109(36): 14574-14579.

13 Jiang X, Kalajzic Z, Maye $P$ et al. Histological analysis of GFP expression in murine bone. J Histochem Cytochem 2005; 53(5): 593-602.
14 Sun L, Peng Y, Sharrow AC et al. FSH directly regulates bone mass. Cell 2006; 125(2): 247-260.

15 Colaianni G, Sun L, Di Benedetto A et al. Bone marrow oxytocin mediates the anabolic action of estrogen on the skeleton. J Biol Chem 2012; 287(34): $29159-29167$.

16 Dragojevič J, Zupan J, Haring G et al. Triglyceride metabolism in bone tissue is associated with osteoblast and osteoclast differentiation: a gene expression study. J Bone Miner Metab 2013; 31(5): 512-519.

17 Ronnebaum SM, Wu Y, McDonough $\mathrm{H}$ et al. The ubiquitin ligase CHIP prevents SirT6 degradation through noncanonical ubiquitination. Mol Cell Biol 2013; 33(22): 4461-4472.

18 Lemos DR, Eisner C, Hopkins $\mathrm{Cl}$ et al. Skeletal muscle-resident MSCs and bone formation. Bone 2015; 80: 19-23.

19 Lee N, Kim DK, Kim ES et al. Comparative interactomes of SIRT6 and SIRT7: implication of functional links to aging. Proteomics 2014; 14(13/14): 1610-1622.

20 Sugatani T, Agapova O, Malluche HH et al. SIRT6 deficiency culminates in low-turnover osteopenia. Bone 2015; 81: 168-177.

21 Barbuto R, Mitchell J. Regulation of the osterix (Osx, Sp7) promoter by osterix and its inhibition by parathyroid hormone. J Mol Endocrinol 2013; 51(1): 99-108.

22 Hong SH, Lu X, Nanes MS et al. Regulation of osterix (Osx, Sp7) and the Osx promoter by parathyroid hormone in osteoblasts. J Mol Endocrinol 2009; 43(5): 197-207.

(c) (i) This work is licensed under a Creative Commons Attribution 4.0 International License. The images or other third party material in this article are included in the article's Creative Commons license, unless indicated otherwise in the credit line; if the material is not included under the Creative Commons license, users will need to obtain permission from the license holder to reproduce the material. To view a copy of this license, visit http://creativecommons.org/licenses/by/4.0/ 